\title{
Relación pedagógica en la educación intercultural: una aproximación desde los profesores mentores en La Araucanía ${ }^{1}$
}

\author{
Katerin Arias-Ortega ${ }^{2}$ \\ ORCID: 0000-0001-8099-0670
}

\section{Resumen}

En Chile la educación intercultural bilingüe (EIB) es una política del Estado que incorpora la enseñanza de la lengua y la cultura de los pueblos indígenas en el currículum escolar. El problema de investigación plantea que la concretización de la EIB en el aula se encuentra limitada por desafíos epistemológicos entre los encargados de implementarla. El artículo expone resultados de investigación que describen la percepción de profesores mentores sobre los factores que afectan la enseñanza de la lengua y la cultura mapuche en la relación pedagógica con el educador tradicional, en tres escuelas situadas en comunidades mapuche en La Araucanía. La metodología es de carácter cualitativo, con un nivel descriptivo, en que se aplicaron entrevistas semidirigidas a los participantes. El análisis de la información se realiza mediante el uso de la técnica de teoría fundamentada. Los resultados muestran que existe una percepción negativa hacia la EIB, al concebirla como una política educativa impuesta por el Estado, lo que limita un quiebre paradigmático de la escuela para la enseñanza de la lengua y la cultura mapuche en el aula. Se concluye que es urgente poner en discusión y cuestionamiento la política de EIB que desde su enfoque funcional aumenta el racismo y prejuicio hacia lo mapuche en la educación escolar. Por ende, es necesario repensar la educación intercultural desde una perspectiva crítica, que surge desde los sujetos que históricamente han sido subalternizados.

\section{Palabras clave}

Relación educativa - Educación intercultural - Prácticas pedagógicas.

1- Agradecimientos a la Vicerrectoría de Investigación y Posgrado de la Universidad Católica de Temuco, Chile, proyecto PROFONDECYT 2019PFKA-05 y al proyecto FONDECYT 1181531.

2- Universidad Católica de Temuco, Temuco, Chile. Contacto: karias@uct.cl.

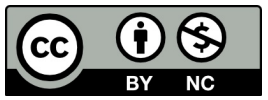




\title{
Pedagogical relationship in intercultural education: an approach from mentor teachers in La Araucanía
}

\begin{abstract}
In Chile, bilingual intercultural education (EIB) is a State policy that incorporates the teaching of the language and culture of indigenous peoples in the school curriculum. The research problem suggests that carrying out EIB in the classroom is limited by epistemological challenges put to those responsible for implementing it. The article presents research results that describe the perception of mentor teachers related to factors that affect the teaching of the Mapuche language and culture in the pedagogical relationship with the traditional educator, in three schools located in Mapuche communities in La Araucania. The methodology is qualitative, with a descriptive level, in which semi-directed interviews were conducted with the participants. The analysis of the information was done by using the grounded theory technique. Results show that there is a negative perception towards $E I B$, when it is conceived as an educational policy imposed by the State, which limits a paradigmatic breakdown of the school for teaching Mapuche language and culture in the classroom. Conclusion is that there is an urgent need to discuss and question the EIB policy, which due to its functional focus increases racism and prejudice towards the Mapuche in school education. Therefore, it is necessary to rethink intercultural education from a critical perspective, which arises from subjects who have historically been subordinated.
\end{abstract}

\section{Keywords}

Educational relationship - Intercultural education - Pedagogical practices.

\section{Introducción}

El propósito de la educación intercultural bilingüe (EIB) en Chile es la enseñanza de la lengua y la cultura indígena en el marco de la educación escolar (CHILE, 2017). Esto se asume como una forma de reparación histórica con los pueblos indígenas, respecto de una educación formal que desconoce los saberes y conocimientos educativos indígenas en el currículum escolar y que ha sido la responsable por la baja vitalidad lingüística y debilitamiento de la identidad sociocultural indígena. La operacionalización de la EIB en escuelas de La Araucanía se da en el marco del establecimiento de una relación educativa entre un profesor mentor y un educador tradicional. El profesor mentor es definido desde el Ministerio de Educación (MINEDUC), como el profesional de la educación encargado de apoyar y monitorear el desenvolvimiento del educador tradicional en la sala de clases (CHILE, 2011). Mientras que el educador tradicional es definido como Kimche (sabio) y en general es validado por la comunidad por ser poseedor de saberes y conocimientos 
educativos propios, deseables de transmitir a las nuevas generaciones mapuches en la escuela, mediante sus intervenciones pedagógicas (CHILE, 2011). La relación educativa entre el profesor mentor y el educador tradicional implica un proceso de interacción comunicativa, desde dos marcos epistemológicos culturalmente distintos (occidental e indígena), que entran en vinculación durante la enseñanza de la lengua y la cultura (FILLOUX, 1984; HOUSSAYE, 1993; POSTIC, 2001; HENON, 2012). Dichos procesos de interacción son posibles de estudiar de acuerdo con el modo en el que surgen y los contenidos que abordan, según la percepción de los sujetos implicados en la relación educativa. Lo anterior permite estudiar las complejas relaciones que surgen entre personas, grupos o instituciones de sociedades y culturas diferentes que entran en interacción en el marco de la implementación de la EIB (CHOBAUX, 1972; POSTIC, 2001).

El artículo tiene como objetivo exponer los resultados de investigación sobre la relación educativa en la educación intercultural, desde la aproximación de los profesores mentores, en La Araucanía.

\section{Marco teórico}

La EIB en Latinoamérica se ha impulsado desde las demandas de los grupos minoritarios con sus lenguas, sus culturas, sus visiones de la historia, quienes promueven movimientos reivindicativos de sus espacios sociales y territoriales, para desarrollarse y transmitir a las nuevas generaciones su patrimonio histórico (LAVOREL, 2015). Estas demandas se inscriben en la perspectiva de revitalización social, cultural y lingüística, como lo expresan algunas constituciones políticas en diversos países de Latinoamérica y/o a través de decretos de EIB, que promueven una relación más equitativa y armónica entre la sociedad hegemónica y los pueblos indígenas, tal cómo se presenta en la Tabla 1.

En la tabla 1 se observa que estas reivindicaciones de los pueblos indígenas, plasmados a través de leyes, decretos y políticas educativas, contribuyen en la evaluación crítica de los Estados a nivel político; y de los sistemas escolares, para identificar los principales problemas que enfrenta la población indígena. Entre las problemáticas destacan: 1) la exclusión sistemática de los conocimientos y lenguas indígenas en la institución escolar, tanto en el currículum escolar como en las prácticas pedagógicas de los docentes (CAJETE, 1994; BATTISTE; HENDERSON, 2000); 2) las verdades únicas del conocimiento escolar que oprimen a los estudiantes de grupos minoritarios (étnicos, culturales e indígenas) mediante la violencia simbólica, la desvalorización involuntaria de sus creencias y sus valores culturales (FERRÃO, 2010; SANTOS, 2010); 3) los pensamientos y voces indígenas (pensamientos políticos) son silenciados y dominados por símbolos culturales occidentales (SHIZHA, 2008); 4) la visión hegemónica del mundo persiste en las construcciones del conocimiento científico utilizadas en los sistemas educativos escolares (FERRÃO, 2010); 5) la escolarización es un legado de la hegemonía occidental que puede llegar a una visión alienante del mundo para los indígenas (MAMPAEY; ZANONI, 2015); 6) los profesores son proveedores de un conocimiento universalmente verdadero y niegan cualquier otra episteme, por ejemplo de los pueblos indígenas (ZAMBRANA, 2014); y 7) los profesores se conciben como custodios conservadores del conocimiento dominante, manifestando actitudes negativas hacia el conocimiento indígena. En base al reconocimiento 
de dichas problemáticas, se proponen y promueven formas y estrategias de dar respuesta a una educación pertinente social, cultural, lingüística y territorialmente, para los pueblos indigenas. De este modo, la EIB debe permitir un aprendizaje mutuo y la posibilidad de generar una universalidad, producto de un diálogo intercultural entre sujetos de culturas y sociedades diferentes (ABDALLAH-PRETCEILLE, 2011). Desde un enfoque crítico, la EIB va más allá de la incorporación de contenidos educativos indígenas al currículum escolar, más bien implica promover en la educación escolar la coexistencia de sujetos pertenecientes a sociedades y culturas diferentes, fortaleciendo la convivencia sustentada en el diálogo y en un espacio común que debiese de estar basado en el respeto y las relaciones de igualdad (FERRA0, 2010). En Chile el marco normativo y curricular plantea lineamientos que sustentan la EIB, tal como se evidencia en la tabla 2.

Tabla 1- Políticas educativas, leyes y decretos sobre pueblos indígena

\begin{tabular}{|c|c|}
\hline País & Número de la Ley \\
\hline Argentina & Ley №20206 del año 2006 \\
\hline Bolivia & Ley № 070 del año 2010 \\
\hline Brasil & $\begin{array}{l}\text { Ley №3394 del año } 1996 \\
\text { Ley № } 10172 \text { del año } 2010\end{array}$ \\
\hline Ecuador & Ley Orgánica de Educación Intercultural del año 2011 \\
\hline México & $\begin{array}{l}\text { Ley Orgánica de Derechos Lingüísticos de los Pueblos Indígenas del } 2003 \\
\text { Ley Federal que previene y elimina la discriminación del año } 2003 \\
\text { Programa de Desarrollo de Pueblos Indígenas del año } 2009\end{array}$ \\
\hline Paraguay & $\begin{array}{l}\text { Ley №28/92 del año } 1992 \\
\text { Leyes de Mantención de las Lenguas Vernáculas }\end{array}$ \\
\hline Perú & Ley №28.044 del año 2003 \\
\hline Colombia & $\begin{array}{l}\text { Decreto } 1088 \text { de } 1993 \text { Crea las asociaciones de Cabildos y/o Autoridades Tradicionales indígenas } \\
\text { Decreto } 804 \text { de } 1995 \text { Regula la atención educativa para las comunidades étnicas } \\
\text { Decreto } 1745 \text { de } 1995 \text { Declara el Derecho de Propiedad colectiva de las tierras de las comunidades negras } \\
\text { Decreto } 2164 \text { de } 1995 \text { Titulación de Tierra a las Comunidades Indígenas } \\
\text { Decreto } 2249 \text { de } 1995 \text { Comisión Pedagógica de Comunidades Negras } \\
\text { Decreto } 1396 \text { de } 1996 \text { Crea la Comisión Nacional de Territorios Indígenas y el Programa Especial de Atención a } \\
\text { Pueblos Indígenas } \\
\text { Decreto } 1397 \text { de } 1996 \text { Crea la Comisión Nacional de Territorios Indígenas y la mesa Permanente de Concertación con } \\
\text { los Pueblos y las Organizaciones Indígenas } \\
\text { Decreto } 1320 \text { de } 1998 \text { Reglamenta la Consulta Previa con las comunidades indígenas y negras para la explotación de } \\
\text { recursos naturales dentro de su territorio } \\
\text { Decreto } 982 \text { de } 1999 \text { Comisión para el Desarrollo Integral de la Política Indígena }\end{array}$ \\
\hline Guatemala & $\begin{array}{c}\text { Constitución de la República de Guatemala 1995, declara en el artículo } 65,66,67 \text { y } 68 \text { protección a los grupos } \\
\text { indígenas, sus tierras y comunidades de los grupos originarios del territorio. }\end{array}$ \\
\hline
\end{tabular}

Fuente: Adaptado de Becerra, Bolomey y Meli (2019) y Del Pino y Ferrada (2019). 
Tabla 2- Marcos Normativos y Curriculares que sustentan la ElB en Chile

\begin{tabular}{ccc}
\hline & $\vdots$ & Convenio 169 sobre Pueblos Indígenas \\
& $\vdots$ & Ley Indígena No 19.253 \\
& $\vdots$ & Decreto SLI No280 (Marco curricular Sector Lengua Indígena). \\
Aspectos normativos & $\vdots$ & Decreto Exento N²960 Aprueba Planes y Programas de Educación Básica Asignatura Lengua \\
Indígena.
\end{tabular}

Fuente: Elaboración propia según datos del MINEDUC de 2010 a 2017.

En una perspectiva contemporánea sostenemos que la EIB no debe ser destinada sólo a indígenas, sino más bien debe ser algo universal, que implique a todos los sujetos pertenecientes a la sociedad en su conjunto (SANTOS, 2010). De esta manera, las prácticas educativas se deben desarrollar en un entorno interactivo, con la implicancia de todos los sujetos que allí participan. Así, el rol de la EIB en contextos indígenas implica necesariamente combatir el prejuicio para construir nuevas bases de interrelación entre las personas que se encuentran compartiendo una misma realidad, es decir, una educación intercultural para todos. La EIB en contexto mapuche implica considerar ambas lógicas de pensamiento, sin segregar uno de otro, o transformarse en un aspecto aislado.

\section{Metodología}

En el estudio nos situamos desde la investigación cualitativa, para comprender la implementación de la EIB según la perspectiva de los profesores mentores (FORTIN, 2010; GAUTHIER; BOURGEOIS, 2016). La muestra corresponde a tres profesores mentores que se desempeñan en escuelas situadas en comunidades mapuches en La Araucanía. El instrumento de recolección de datos es la entrevista semidirigida. La técnica de análisis empleada es la teoría fundamentada (GAUTHIER; BOURGEOIS, 2016). Con el propósito de identificar las entrevistas de profesores mentores durante su proceso de codificación, se utiliza como nomenclatura los siguientes símbolos: 1) PM (profesor mentor), el número de entrevista y la sigla correspondiente a la territorialidad del entrevistado. También, se incorpora el número en el cual se encuentra la expresión del profesor mentor en la unidad hermenéutica de atlas ti con un paréntesis cuadrado. Por ejemplo: (PM1L [233:233]). 


\section{Resultados}

Los resultados que emergen del análisis de las entrevistas aplicadas a los profesores mentores nos permiten constatar que existen factores que limitan la implementación de la EIB. Además, identificamos factores que fortalecen dicha implementación y desafíos que surgen para mejorarla en tres escuelas de La Araucanía. Así, los testimonios de los profesores mentores se constituyen en una base de conocimiento que aporta con evidencias empíricas, explicitando los contenidos conceptuales, procedimentales y actitudinales que inciden en la implementación de la EIB. La organización de los resultados se realiza en relación a dos categorías centrales que emergen con una mayor recurrencia en los testimonios de los profesores mentores. Las categorías centrales y sus recurrencias se consignan en la siguiente tabla.

Tabla 3- Categorías centrales según testimonio profesores mentores

\begin{tabular}{|c|c|c|}
\hline Categoría & Recurrencias & Porcentaje \\
\hline Desarrollo de la implementación de la EIB & 139 & $66 \%$ \\
\hline $\begin{array}{l}\text { Desafíos en la implementación de la EIB, en el proceso de enseñanza- } \\
\text { aprendizaje del mapunzugun }\end{array}$ & 72 & $34 \%$ \\
\hline Total & 211 & $100 \%$ \\
\hline
\end{tabular}

Fuente: Elaboración propia con apoyo del programa Atlas ti.

\section{Desarrollo de la relación educativa}

En la tabla 3, la categoría desarrollo de la relación educativa entre el profesor mentor y el educador tradicional se organiza en dos subcategorías. La primera subcategoría refiere a limitaciones que emergen desde el testimonio de los profesores mentores respecto del desarrollo de la relación educativa. Esta subcategoría obtiene 88 recurrencias, equivalente a un 63\%. Así, desde el testimonio de los profesores mentores se indica que las limitaciones en la implementación de la EIB están asociadas a la relación educativa que se establecen con el educador tradicional, en la que emergen los siguientes códigos: 1) el prejuicio que emerge hacia el ser mapuche por parte de los profesores mentores. Este código obtiene 38 recurrencias, equivalente a un 43,1\%. El prejuicio se expresa a través de frases como: son pobres, deben preocuparse por salir del campo, les cuesta aprender. Estas expresiones develan como desde la perspectiva de los profesores mentores se asume a la familia de los estudiantes mapuches, caracterizada por la pobreza y bajo nivel cognitivo; 2) la resistencia del profesor mentor para el desarrollo de un trabajo colaborativo con el educador tradicional, durante la implementación de la EIB. Este código obtiene 23 
recurrencias, equivalente a un 26,1\%. Esta resistencia se oculta bajo premisas como el educador tradicional no colabora, no aporta con guias o planificación de clases; 3) la baja escolarización del educador tradicional que limitaría la relación educativa. Este código obtiene 15 recurrencias equivalente a un 17\%. Esta subcategoría se manifiesta mediante frases como: el educador tradicional debería al menos tener octavo año básico, no saben escribir, los niños no entienden su letra; y 4) la desvalorización del mapunzugun por parte del profesor mentor, los padres y los estudiantes, que se manifiesta en los procesos de enseñanza-aprendizaje, mediante expresiones como les será más útil aprender inglés. Este código obtiene 12 recurrencias equivalente a un 14\%.

La segunda subcategoría, refiere a las fortalezas que existen en la relación educativa. Esta subcategoría obtiene 51 recurrencias, equivalente a un 37\% y se relaciona con los siguientes códigos: 1) la confianza del profesor mentor con el educador tradicional para establecer una relación educativa positiva, durante la implementación de la EIB. Este código obtiene 23 recurrencias, equivalente a un 45\%;2) la claridad de los roles y funciones que debe cumplir cada agente educativo en la EIB. Este código obtiene 12 recurrencias, equivalente a un $24 \%$; 3) las posibilidades que otorga la enseñanza del mapunzugun, referida a la incorporación de métodos educativos mapuches en la implementación de la EIB. Este código obtiene 16 recurrencias, equivalente a un 31\%. La primera categoría central y sus subcategorías se presentan en la figura 1.

A continuación, se describe cada subcategoría, exponiendo la percepción del profesor mentor sobre la relación educativa en la EIB en contexto mapuche.

\section{Limitaciones en el desarrollo de la relación educativa}

Las limitaciones en el desarrollo de la relación educativa que emergen en el discurso de los profesores mentores refiere a aspectos que dificultan el aprendizaje del mapunzugun. Entre las limitaciones está la resistencia del profesor mentor para la incorporación del mapunzugun en el proceso educativo, debido al bajo valor social que se le otorga a la lengua. Esta resistencia se conjuga con la emergencia de prejuicios hacia el ser mapuche y la baja escolarización del educador tradicional.

En relación al prejuicio hacia el mapuche, este se refiere a las concepciones negativas preconcebidas por parte del profesor mentor hacia el educador tradicional y estudiante mapuche, sólo por el hecho de su identidad sociocultural. Dichos prejuicios se sustentan en una minorización del mapuche por considerarlo un sujeto que vive en la pobreza y por lo cual presentaría un bajo nivel cognitivo. Es por ello que el profesor mentor desarrolla una actitud paternalista hacia el mapuche, asumiendo el rol de salvador de los estudiantes. Es lo que observamos en el siguiente testimonio: "Son niños que necesitan harta preocupación de parte del profesor, están metidos en la pobreza, [...] falta que salgan del campo a la ciudad y vean otra vida, para salir de la miseria en que viven" (PM1L [13:13]). Desde la perspectiva del profesor mentor, el abandono y desarraigo social, cultural y territorial de la comunidad mapuche es la única forma de salir de la pobreza, porque el campo está asociado a una forma de retraso y malas condiciones de vida. El deseo del profesor mentor sobre la necesidad de que los 
Figura 1- Desarrollo de la relación educativa

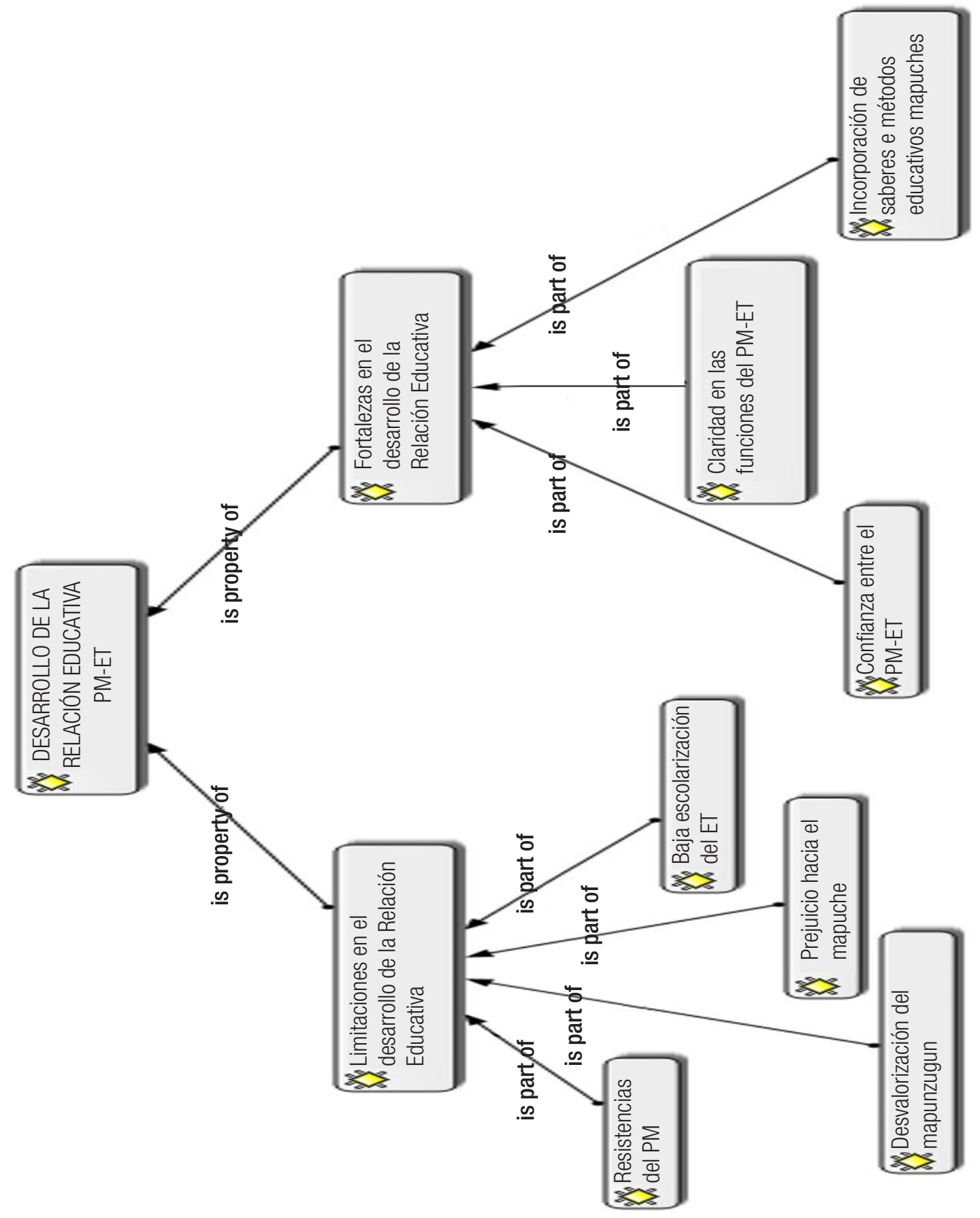

Fuente: Elaboración propia con apoyo del programa Atlas ti. 
estudiantes abandonen el campo se ha empezado a masificar en los padres, quienes progresivamente han comenzado a pensar y asumir que abandonar el campo es la mejor opción para tener un ascenso social en la sociedad dominante.

Otro prejuicio asociado al mapuche refiere a su conflictividad. Esta representación social negativa hacia el mapuche que busca reivindicar su territorio ha sido transmitida, generalmente, a través de los textos de estudio y los medios de comunicación a nivel nacional. En este contexto, un profesor mentor menciona que les comenta a los estudiantes, que no todos los mapuches son conflictivos, que en el territorio donde ellos habitan la conflictividad no existe. Es lo que observamos en el siguiente testimonio: “[...] ellos mismos [los estudiantes], ven en las noticias, cuando sale que los mapuches se toman la tierra, [...] eso les duele a los niños, cuando hablan que los mapuches son aquí, que son problemáticos, que son revolucionarios" (PM1L [62:62]). De acuerdo al testimonio del profesor mentor, evidenciamos que estas manifestaciones se constituyen en actitudes y gestos que se terminan conjugando en la reconfiguración de otras formas inconscientes de transmitir prejuicios hacia el educador tradicional y los estudiantes mapuches.

Respecto a la resistencia de los profesores mentores, esta se manifiesta a través de la negación de aprender el mapunzugun bajo excusas, tales como la existencia de diferentes grafemarios y la no escritura de dicha lengua. Al respecto un profesor mentor señala que "[...] muchas dificultades [he tenido con el mapunzugun], a mí me ha costado, porque no entiendo al tío [educador tradicional] cuando me habla en mapuche, no sé. No, no puedo, no puedo, no puedo aprender el mapunzugun" (PM1L [24:27]). La negación para aprender el mapunzugun, por parte del profesor mentor, se ha constituido en una actitud que los estudiantes han imitado, por eso cuando el educador tradicional los saluda o intenta desarrollar una actividad de aprendizaje es ignorado por los estudiantes. Asimismo, la resistencia del profesor mentor para el aprendizaje del mapunzugun ha influido en su auto-negación para desarrollar un trabajo colaborativo en la gestión de la clase, ya sea, antes, durante y después del desarrollo del proceso de enseñanzaaprendizaje del mapunzugun. En ese sentido, la resistencia del profesor mentor se pone de manifiesto en el trabajo de planificación de las actividades de aprendizaje y la elaboración de material educativo para la implementación de la EIB. De esta manera, es el profesor mentor quien asume unilateralmente la responsabilidad de planificar las actividades de aprendizaje, impidiendo los aportes que pudiese hacer el educador tradicional. Es lo que evidenciamos en el siguiente testimonio de un profesor mentor:

El trabajo técnico pedagógico es mío, yo planifico las actividades de aprendizaje, selecciono los materiales educativos y decido qué contenidos trabajaremos. Lo hago porque el educador tradicional no sabe hacer eso, y hacerlo con él [planificación escolar] me tomaría mucho más tiempo, porque tendría que enseñarle. (PM2W [51:51]).

En el testimonio del profesor mentor, observamos que la resistencia para desarrollar el trabajo colaborativo es justificada por la falta de competencias pedagógicas del educador tradicional para desarrollar una planificación escolar. Es por ello que asumen que para él es más eficiente y eficaz realizar el trabajo de gestión de la clase de manera 
independiente. Sin embargo, desde las orientaciones dadas por el EIB del MINEDUC, se propone el desarrollo de un trabajo colaborativo en los procesos de enseñanza-aprendizaje del mapunzugun, para apoyar el trabajo pedagógico de ambos y generar una relación educativa que posibilite el aprendizaje de los estudiantes de manera pertinente. Así, la resistencia del profesorado al trabajo de acompañamiento es un denominador común en su discurso, proceso en el que se termina culpabilizando al educador tradicional y sus carencias didácticas y pedagógicas como los factores que limitan el desarrollo de una relación educativa sustentada en la colaboración. De este modo, los profesores mentores asumen que los educadores tradicionales no tienen las competencias para aportar a los procesos de enseñanza-aprendizaje de la lengua y la cultura mapuche en la educación escolar. Esta realidad constituye uno de los principales problemas que enfrentan los educadores tradicionales en la relación educativa con el profesor mentor. Al respecto un profesor mentor menciona de manera literal que "Él [educador tradicional] no tiene la capacidad de trabajar con los niños, la clase es un desorden. Yo he observado que no sabe, no domina a los estudiantes, no tiene compromiso, no sabe enseñar la lengua y escribe pésimo" (PM1L [31:31]). En relación al testimonio del profesor mentor observamos que se atribuyen al educador tradicional características negativas, concibiéndolo como un sujeto incapaz de desarrollar una situación de aprendizaje, pertinente y adecuada en la educación escolar.

En consecuencia, se asume al educador tradicional como alguien que no sabe y no domina las competencias profesionales, por lo que no desarrolla una intervención pedagógica adecuada. Al respecto, un profesor mentor menciona que "[...] la baja escolarización del educador tradicional incide en las prácticas pedagógicas porque no es capaz de planificar o desarrollar una clase motivadora para los estudiantes. Él carece de una estructura coherente, para abordar los distintos objetivos de aprendizaje" (PM3N [19:19]). Desde el testimonio de los profesores mentores se asume que la baja escolarización del educador tradicional es el principal factor que afecta sus prácticas pedagógicas, es una limitante de la relación educativa e influye en la disposición de los estudiantes para aprender el mapunzugun. Respecto de ello, un profesor mentor menciona que:

\footnotetext{
No, sus clases de mapunzugun no las encuentro motivadoras, porque les dice a los niños que escriban una palabra en mapunzugun, y no es así. Le digo tío [educador tradicional] hagamos la clase de esta manera [más didáctica, con juegos], pero no, no quiere, me dice [profesor mentor] déjeme a mí hacer la clase de mapunzugun. (PM1L [43:43]).
}

En el testimonio de los profesores mentores develamos que, para la totalidad de ellos es imprescindible que el educador tradicional cumpla con ciertas condiciones, que facilitarían establecer una relación educativa positiva, caracterizada por la colaboración mutua en el proceso de enseñanza-aprendizaje del mapunzugun. Es así como las condiciones que debiese tener el educador tradicional, según los profesores son: 1) mayor nivel de escolarización, ya sea Educación Media Completa o idealmente Educación Superior, dominio pedagógico y didáctico referido a saber planificar, elaborar material 
didáctico y dominio de grupo (PM1L [23:23]); 3) dominio de estrategias y habilidades para la enseñanza del mapunzugun (PM3N [17:17]); 4) sentirse mapuche, porque cuando uno se siente mapuche tiene ese conocimiento cultural y ese kimün (conocimiento), que puede entregar a los niños en la escuela (PM2W [15:15]); 5) querer enseñar el mapunzugun (PM2W [17:17]); 6) conocer la vida que tienen los mapuches, producto de su educación familiar (PM1L [23:23]); y 7) compromiso [por parte del educador tradicional], para enseñar el mapunzugun en la escuela (PM2W [16:16]). Según el testimonio de los profesores mentores, el educador tradicional debiera cumplir con dichas condiciones para establecer una relación educativa colaborativa en los procesos de enseñanza-aprendizaje del mapunzugun. Asimismo, emerge del testimonio de los profesores mentores, que ellos también debiesen cumplir con condiciones para poder participar en la implementación de la EIB. Dichas condiciones son: 1) ser profesor intercultural lo que le permita la articulación de los saberes y conocimientos educativos mapuches y escolares; 2) ser capaz de desarrollar un trabajo de colaboración con el educador tradicional en el aspecto técnico pedagógico (PM3N [15:15]); 3) conocer la lengua vernácula para facilitar el desarrollo de actividades de aprendizaje más didácticas y ser capaz de llevar el liderazgo pedagógico en el aula (PM3N [27:27]); 4) dominio de estrategias didácticas para el proceso de enseñanza de una segunda lengua (PM3N [28:28]); y 5) disposición y motivación para trabajar en equipo de co-enseñanza (PM2W [17:17]).

En consecuencia, en el testimonio de los profesores mentores se plantea la necesidad de avanzar progresivamente en procesos de capacitación y apoyo técnico pedagógico, tanto para ellos, como para los educadores tradicionales. De acuerdo con los testimonios de los profesores mentores, constatamos que son conscientes de las limitaciones y fortalezas que enfrentan en el desarrollo de la relación educativa. No obstante, aún no son capaces de visibilizar actitudes racistas y prejuiciadas hacia el mapuche, las que se constituyen en factores que tensionan la relación educativa.

Respecto a la desvalorización del mapunzugun por parte de los profesores mentores, los padres y los estudiantes, ello se refleja en una baja valoración e importancia que se le atribuye a la lengua mapuche, cuestión que se ve expresa cuando se asume el mapunzugun como una lengua poco útil en el proceso educativo escolar. En el testimonio de los profesores mentores esta desvalorización hacia la lengua mapuche se expresa a través de la resistencia para modificar las prácticas pedagógicas monolingües en español, aun cuando se estén desarrollando las clases de lengua y cultura mapuche en el aula. Es lo que señaló un profesor mentor en su testimonio "No sé hablar mapunzugun, no lo entiendo [...], acá hacemos las clases en castellano y el tío traduce las palabras [...], los estudiantes y yo [profesor mentor], no le hemos tomado el gusto o el sentido al mapunzugun" (PM1L [45:45]). De acuerdo al relato del profesor mentor, evidenciamos que la desvalorización del mapunzugun se refiere al desconocimiento y negación de él para aprender el mapunzugun. Esto ha generado que las clases se focalicen en la traducción de palabras y el aprendizaje de vocabulario, lo que ha traído como consecuencia un bajo interés por parte de los estudiantes y un rechazo de estos para aprender el mapunzugun.

También evidenciamos en el relato de los profesores mentores que no sólo ellos son quienes no le otorgan valor al mapunzugun, sino que también, es una práctica 
habitual de los padres, quienes rechazan y cuestionan el por qué los estudiantes deben aprender el mapunzugun, si su utilidad para el desarrollo de los procesos de aprendizaje es básica. Esto se remarca en el siguiente testimonio de un profesor mentor "Igual la gran mayoría de los papás acá [en esta escuela], dicen, tía está bien que se enseñe la lengua mapunzugun, pero mi hijo tiene que aprender más inglés, porque cuando se van a la ciudad, ya no le pasan mapunzugun" (PM1L [19:19]). En ese sentido, los profesores mentores agregan a su testimonio que este tipo de actitudes de rechazo de los padres hacia la enseñanza del mapunzugun son actitudes percibidas por sus hijos, quienes asumen una postura de negación y desmotivación para el aprendizaje de la lengua mapunzugun. Esto se manifiesta en una actitud negativa hacia las actividades de aprendizaje que el educador tradicional desarrolla. Respecto a ello, en un testimonio se señala que: “[...] a los niños no les interesa aprender su lengua. Una vez hicimos una encuesta de quiénes querían aprender la lengua y ninguno levantó la mano [...], cuando se quería dar un contenido en mapunzugun los niños lo rechazaban, eso es penoso" (PM3N [25:25]). Según el testimonio de los profesores mentores, observamos que la desvalorización del mapunzugun es una práctica tanto del profesor mentor, como de los padres y los estudiantes.

\section{Fortalezas profesionales y personales en la relación educativa}

Las fortalezas profesionales y/o personales en la implementación de la EIB están asociadas a: 1) la confianza mutua para desarrollar los procesos de enseñanza-aprendizaje del mapunzugun; 2) la claridad en las funciones y responsabilidades que cada uno asume para el desarrollo de la enseñanza del mapunzugun; y 3) la incorporación de saberes y métodos educativos mapuches en las prácticas pedagógicas en la EIB.

Respecto a la confianza en la relación educativa los profesores mentores declaran que han logrado establecer lazos de confianza mutua con el educador tradicional. En relación a ello, un profesor mentor señala que “[...] esta confianza que establezco con el educador tradicional se refleja cuando él es capaz de conversar conmigo y contarme sus propias experiencias en el trabajo pedagógico" (PM3N [100:100]). Este tipo de confianza les otorga la oportunidad de conocerse mutuamente y de esta manera incorporar esas vivencias en las prácticas pedagógicas. De acuerdo a lo anterior, un profesor mentor menciona que "[...] cuando yo no sé algo, soy sincero y se lo digo al educador tradicional. Lo mismo sucede cuando él no sabe algo, me lo dice y en conjunto buscamos una solución” (PM3N [42:42]). De acuerdo al testimonio, la confianza que logran establecer con el educador tradicional es mutua y se constituye en una fortaleza de la relación educativa, ya que ambos agentes educativos serían capaces de evidenciar los desconocimientos y dificultades que tienen para abordar ciertos contenidos educativos mapuches en la EIB.

También constatamos que una de las principales fortalezas que han logrado establecer el profesor mentor con el educador tradicional está referida a la claridad de sus funciones y sus responsabilidades. Es así como cada uno de los agentes educativos conoce las actividades que debe desarrollar y los tiempos de intervención de cada 
uno. En ese sentido, un profesor mentor relata que “[...] el educador tradicional es el encargado de dar el inicio a la clase, de explicar el contenido cultural y de realizar el cierre de la clase" (PM2W [12:12]). Así, las principales funciones del educador tradicional en la implementación de la EIB refieren a: 1) traducción de palabras o frases del castellano al mapunzugun (PM3N [68:68]); 2) realizar el nüxamkan (conversación), para explicar el contenido cultural mapuche y monitorear el trabajo de los estudiantes (PM2W [47:47]); y 3) realizar actividades artísticas y de artesanía con los estudiantes (PM1L [95:95]). Mientras que los profesores se encargan de: 1) realizar el trabajo técnico pedagógico referido a la planificación de las actividades de aprendizaje; 2) elaborar el material educativo; y 3) realizar la selección de los contenidos educativos mapuches, que se abordan en el proceso de enseñanza-aprendizaje. Esto se ve reflejado en el siguiente testimonio: "Yo soy la persona que planifica la clase, su estructura y quien incorpora el conocimiento más técnico occidental. Él realiza la bajada cultural de los contenidos mapuches" (PM2W [13:13]). Según el testimonio, podemos inferir que para desarrollar las prácticas pedagógicas y la gestión de la clase las responsabilidades están claramente definidas y asumidas, tanto por el profesor mentor como por el educador tradicional.

Desde la perspectiva de los profesores mentores, otra fortaleza de la relación educativa refiere a la posibilidad de incorporar saberes y métodos educativos mapuches a la educación escolar. En efecto, esta incorporación de contenidos mapuches la conciben como una posibilidad para avanzar en el conocimiento y adquisición de mayor vocabulario en mapunzugun, tanto de los estudiantes como de los profesores. En ese sentido, los profesores mentores reconocen que en la relación educativa la incorporación de contenidos educativos mapuches ha permitido profundizar en la importancia de los valores educativos mapuches a todos los actores implicados en el proceso de enseñanza-aprendizaje: profesor mentor, educador tradicional y los estudiantes. Así, reconocen que el respeto, desde la lógica cultural mapuche, es un contenido importante que les permite relacionarse con el medio familiar y comunitario, según las normas y patrones socioculturales propios. Al respecto un profesor mentor señala que: "El respeto desde la lógica cultural mapuche es muy importante, demostrarlo a los mayores, a la naturaleza y el cuidado hacia nuestro planeta" (PM3N [61:61]). En efecto, reconocen que los valores educativos mapuches constituyen contenidos escolares para formar a los estudiantes en la relación del hombre con el medioambiente. Asimismo, señalan que la incorporación de métodos educativos mapuches no solo ha permitido desarrollar procesos de enseñanza-aprendizaje más pertinentes, sino que, además ha permitido aprender conocimientos asociados a la historia local. Al respecto, un profesor mentor señala que "[...] es importante el conocimiento mapuche para la formación de los niños, porque deben saber de dónde vienen, cuáles fueron sus antepasados, la historia, deben conocer qué es lo que pasó [con los mapuches históricamente]" (PM1L [60:60]). De acuerdo al testimonio del profesor mentor, para que los estudiantes conozcan su propia historia se requiere de la utilización de métodos educativos mapuches, como una forma de transmitir los conocimientos, desde su propia lógica. 


\section{Desafíos de la relación educativa}

La categoría desafíos de la relación educativa emerge desde el testimonio de los profesores mentores. Esta categoría alcanza 72 recurrencias, equivalente a un $34 \%$ y se compone de cuatro códigos. El primer código refiere a la negociación de los conocimientos educativos mapuches y escolares en el marco de la relación educativa que establecen los profesores mentores y los educadores tradicionales en la implementación de la EIB. Obtiene 32 recurrencias, equivalente a un 44\%. El segundo código refiere a la incorporación de la toponimia local en los procesos de enseñanza-aprendizaje de la lengua y la cultura mapuche. Este aspecto permite el juego de roles y funciones de los agentes educativos en la implementación de la EIB, facilitando de esta manera que ambos compartan las responsabilidades de que los estudiantes conozcan su contexto social y cultural. Este código obtiene 23 recurrencias, equivalente a un 32\%. El tercer código refiere a la necesidad de implementar procesos de capacitación en el ámbito pedagógico, didáctico y metodológico para los agentes educativos, que les permitan adquirir las herramientas para implementar la EIB de forma eficaz y eficiente. Obtiene 9 recurrencias, equivalente a un 13\%. Finalmente, el cuarto código refiere a la vinculación con el medio familiar y comunitario en los procesos de enseñanza-aprendizaje de la lengua y la cultura mapuche. Obtiene 8 recurrencias, equivalente a un 11\%. La categoría central y sus subcategorías se presentan en la siguiente figura.

Figura 2- Desafíos de la relación educativa profesor mentor y educador tradicional

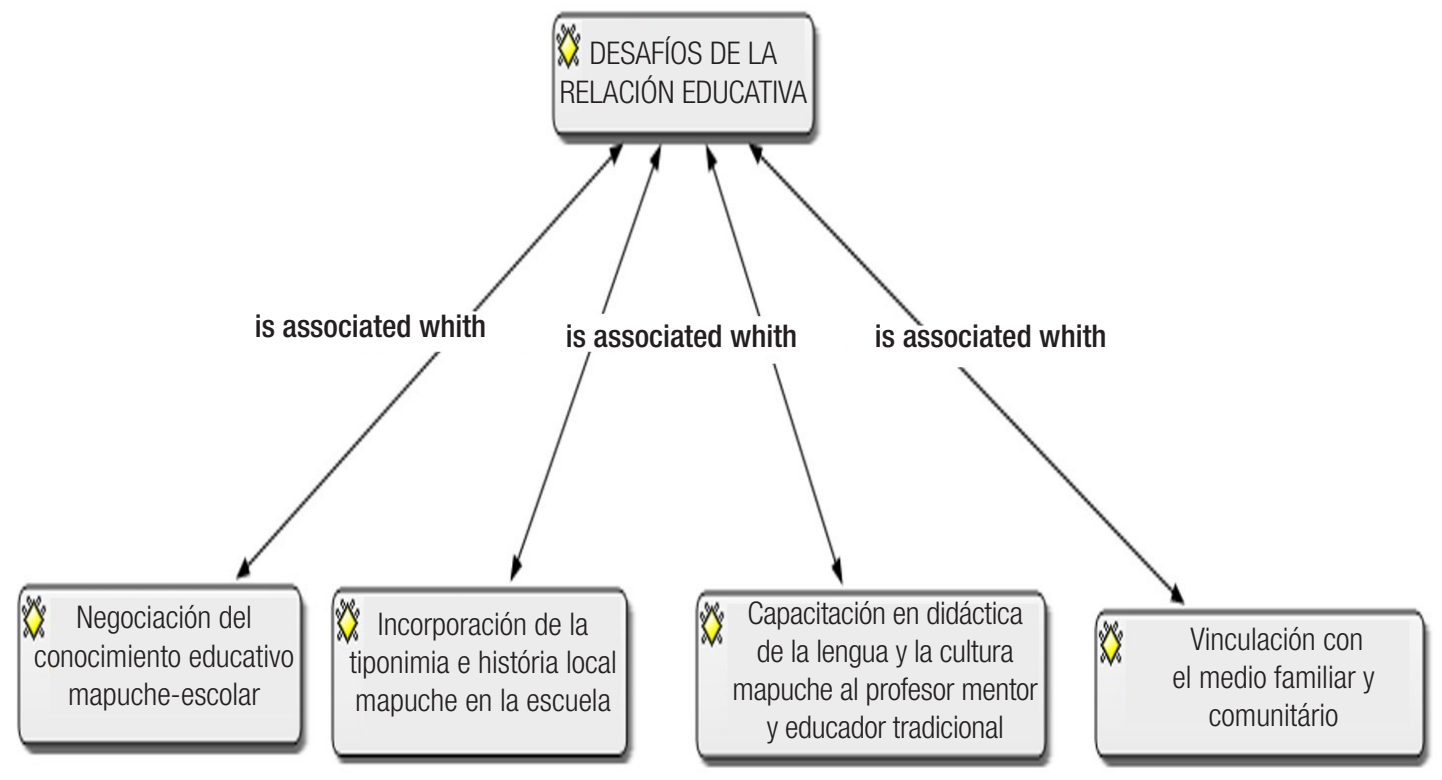

Fuente: Elaboración propia con apoyo del programa Atlas ti. 
Los desafíos de la relación educativa asociados a la capacitación del profesor mentor y el educador tradicional se relacionan con la necesidad de avanzar en el desarrollo profesional en el ámbito didáctico y pedagógico. El propósito es realizar procesos de enseñanza-aprendizaje más didácticos y pertinentes al contexto social y cultural de los estudiantes. Al respecto un profesor mentor señala que "[...] pienso que es necesario capacitar más al profesor mentor [en el dominio de la lengua vernácula] porque se supone [que él es quien] tiene que llevar el liderazgo [pedagógico] dentro de la sala [de clases]" (PM3N [27:27]). Así, la posibilidad de capacitarse en el mapunzugun, es un desafío para establecer una relación educativa de colaboración más eficiente con el educador tradicional. Además, manifiestan la urgencia que el educador tradicional y el profesor mentor se capaciten en estrategias metodológicas para la enseñanza de segundas lenguas.

En la perspectiva de los profesores mentores, otro desafío a lograr en la relación educativa es un compromiso, implicación y vinculación efectiva con el medio familiar y comunitario. Para ello, asumen que es necesario desarrollar los procesos de enseñanzaaprendizaje fuera de la sala de clases. Al respecto un profesor mentor señala que: "A nosotros nos falta salir a terreno, ir a visitar a un logko a la comunidad, esas actividades aparecen en los programas de estudio, pero no lo hemos realizado" (PM3N [38:38]). De acuerdo con ello, el profesor mentor agrega que este tipo de prácticas de vinculación con el medio familiar, le generaría la posibilidad de establecer una relación educativa más cercana, no sólo con el educador tradicional, sino también con la comunidad educativa en general. Asimismo, podrían establecer conversaciones con las autoridades mapuches para conocer sus funciones y roles, de modo tal que se constituyan en actividades que les permitan a ellos y a los estudiantes profundizar en el conocimiento educativo mapuche.

En lo concerniente al desafío de la negociación del conocimiento mapuche y occidental, implica establecer espacios para formalizar los saberes y conocimientos educativos mapuches en la educación escolar. Asimismo, reconocen que es perentorio discutir y negociar con el educador tradicional los métodos educativos mapuches que se utilizarán en el proceso de enseñanza-aprendizaje del mapunzugun para decidir en conjunto las formas de incorporar los conocimientos educativos mapuches en las prácticas pedagógicas. En este aspecto, un profesor mentor relata que: “[...] debemos avanzar y ser capaces de aunar criterios, ya sea en la incorporación de los contenidos educativos mapuches como en la pronunciación del mapunzugun, esto es lo único que nos falta, porque los otros aspectos están bien definidos" (PM2W [29:29]). Es así como la negociación la conciben como una estrategia para establecer una relación educativa adecuada entre ambos, teniendo como objetivo común el éxito escolar y educativo de los estudiantes. De este modo, reconocen que las tensiones que emergen en la relación educativa están asociadas a desacuerdos epistemológicos respecto de cómo concebir la enseñanza desde perspectivas que históricamente han sido antagónicas, es decir, desde la educación familiar mapuche y escolar. De esta manera, la negociación se concibe como una forma de aunar criterios para establecer actividades y situaciones de aprendizaje para los estudiantes. Sin embargo, los profesores mentores reconocen que este trabajo de negociación es progresivo y se va construyendo con el tiempo, en la medida que se van estableciendo relaciones educativas de confianza, respeto y legitimidad con el otro. 
Finalmente, el último desafío que manifiestan los profesores mentores está relacionado con la incorporación de conocimientos educativos mapuches sobre la toponimia e historia local, esto como una estrategia que permitiría a los estudiantes conocer el medio sociocultural en el cual viven. Al respecto, un profesor mentor relata que "[...] es necesario que los estudiantes conozcan los nombres de cada localidad, su sentido y significado, desde la lógica cultural mapuche" (PM3N [98:98]). Esto como una forma de establecer una relación más consciente con su medioambiente.

\section{Puntos para concluir}

Los resultados de investigación nos permiten concluir que existe por parte de los profesores mentores una percepción negativa hacia la EIB, al concebirla como una política impuesta por el Estado. Esta política educativa obliga a los profesores mentores a asumir la enseñanza de la lengua y la cultura mapuche en colaboración con el educador tradicional, sin siquiera estar preparados profesionalmente para esta actividad. Lo anterior, debido a su propio desconocimiento de los saberes y conocimientos educativos mapuches, producto de su formación inicial docente caracterizada por la hegemonía del conocimiento eurocéntrico occidental. Además, reconocen que carecen de competencias disciplinares para la enseñanza de segundas lenguas. Según los profesores mentores, otro problema que afecta negativamente la implementación de la EIB tiene relación con la baja escolarización del educador tradicional. Esto, porque deben destinar acciones para explicar al educador tradicional elementos técnicos como corregir la redacción y ortografía de sus actividades propuestas. En consecuencia, los resultados de investigación nos permiten constatar algunas limitaciones para concretizar la EIB en la institución escolar. Por ejemplo, una percepción prejuiciada hacia el indígena en la figura del educador tradicional, como alguien pobre y con bajo nivel cognitivo. Esto está relacionado con una reproducción social y transmisión de estereotipos a las nuevas generaciones de indígenas y no indigenas, que, en palabras de Lander (2000), están asociadas a esta idea imperante de racismo explícito y encubierto presente en la sociedad y reflejado en prácticas educativas de algunas escuelas. También constatamos que existe una resistencia para el trabajo en colaboración y acompañamiento lo que no permite avanzar en procesos de reflexión y cuestionamiento del propio rol docente para la implementación de la EIB. Sostenemos que para revertir estas problemáticas es necesario que se avance en el reconocimiento y valorización de la lengua vernácula, tanto a nivel de la escuela, padres como estudiantes, visibilizando su riqueza social, cultural y el fortalecimiento de habilidades cognitivas al adquirir un bilingüismo aditivo. Esto permitiría reforzar la identidad social, cultural y el desarrollo de una inteligencia emocional en los estudiantes, fuertemente enraizado a su marco social y cultural propio en vinculación con el conocimiento escolar.

También constatamos fortalezas que se constituyen en instancias que podrían aportar a una efectiva concretización de la EIB en la institución escolar. Tales como: 1) las posibilidades que otorga la enseñanza del mapunzugun referida a la incorporación de métodos educativos mapuches y la incorporación de valores educativos, para la formación de personas; 2) la articulación de saberes y conocimientos educativos mapuche y escolares implica una negociación en la propia interacción que se establece entre el profesor mentor 
y el educador tradicional; y 3) la capacitación y la vinculación con el medio favorecería un trabajo más contextualizado con las necesidades propias de los estudiantes.

En conclusión, los indígenas deben participar en la vida de la sociedad hegemónica, y la sociedad hegemónica debe conceder a los indígenas el derecho a preservar su lengua y su tradición cultural. La incorporación de la lengua y las tradiciones culturales indígenas en la educación escolar es un proceso que a largo plazo contribuirá en el enriquecimiento de todos los estudiantes, generando un mayor vínculo con su comunidad. Esto en las prácticas pedagógicas permite tomar en cuenta las realidades y las necesidades de los estudiantes, según su contexto sociocultural. De esta manera, es posible formarlos como ciudadanos interculturales capaces de vivir juntos con el otro, sin prejuicios en la escuela. Para esto es importante comprender y cuestionar ciertos conceptos y procesos en las relaciones étnicas como la discriminación y el racismo. Esto permitiría el desarrollo de competencias interculturales tanto en el profesor mentor como en el educador tradicional, lo que permitiría una articulación en las prácticas pedagógicas del saber indigena y escolar, en tanto se constituyen en ensambles de saberes, saber ser y saber hacer, como una base sólida para repensar la formación de los niños en la institución escolar, desde un enfoque educativo intercultural.

\section{Referencias}

ABDALLAH-PRETCEILLE, Martine. La pedagogie interculturelle: entre multiculturalisme et universalisme. Linguarum Arena, Porto, v. 2, p. 91-101, 2011.

BATTISTE, Marie; HENDERSON, James. Protecting indigenous knowledge and heritage: a global challenge. Saskatoon: SK - Purich, 2000.

BECERRA, Rukmini; BOLOMEY, Carlos; MELI, Daniela. Teaching indigenous languages in Chile: perceptions of traditional educators. International Journal of Bilingual Education and Bilingualism, Reino Unido, p. 1-19, 2019.

CAJETE, Gregory. Look to the mountain: an ecology of indigenous education. Durango: Kivaki, 1994.

CHILE. Ministerio de Educación. Programa de Educación Intercultural Bilingüe. Consulta a los pueblos indígenas informe de resultados: función docente del educador(a) tradicional para la implementación del sector de lengua indígena. Santiago de Chile: Ministerio de Educación, 2011.

CHILE. Ministerio de Educación. Programa de Educación Intercultural Bilingüe 2010-2016. Santiago de Chile: Ministerio de Educación, 2017.

CHOBAUX, Jacqueline. Étude de la relation éducative: quelques réflexions méthodologiques. Revue française de sociologie, Paris, n. 13, p. 94-111, 1972.

DEL PINO, Miguel; FERRADA, Donatila. Construction of educational knowledge with the Mapuche community through dialogical-kishu kimkelay ta che research. Educational Action Research, Reino Unido, n. 27, p. 414-434, 2019. 
FERRÃO, Marie. Educación intercultural en América Latina: distintas concepciones y tensiones actuales. Revista Estudios Pedagógicos, Valdivia, n. 2, p. 333-342, 2010.

FILLOUX, Jean. La relation educative. Revista Mexicana de Sociología, México, DC, n. 46, p. 369-373, 1984.

FORTIN, Marie. Fondements et étapes du processus de recherche. Montréal: Chenelière Éducation, 2010.

GAUTHIER, Benoit; BOURGEOIS, Isabelle. Recherche sociale de la problématique à la collecte des données. Québec: Presses de l'Université du Québec, 2016.

HENON, Sophie. Percevoir, comprendre et analyser la relation éducative: identification de schèmes d'action y transformation de l'habitus relationnel. Mont Saint Aignan: UFR Sciences de l'Homme et de la Société, 2012.

HOUSSAYE, Jean. La pédagogie, une encyclopédie pour aujourd'hui. Paris: ESF, 1993.

LANDER, Edgar. La colonialidad del saber: eurocentrismo y ciencias sociales. Perspectivas latinoamericanas. Buenos Aires: Consejo Latinoamericano de Ciencias Sociales, 2000.

LAVOREL, Sabine. La revitalisation des langues amérindiennes en Amérique Latine. Revue Internationale, Montreal, p. 2-25, 2015. Disponible en: <https://www.sens.public.org/spip.php?>. Acceso en: 24 mzo. 2019.

MAMPAEY, Jelle; ZANONI, Patrizia. Reproducing monocultural education: ethnic majority staff's discursive constructions of monocultural school practices. British Journal of Sociology of Education, Reino Unido, v. 37 , n. 7, p. 928-946, 2015.

POSTIC, Marcel. La relation éducative. Paris: Presses Universitaires de France, 2001.

SANTOS, Boaventura. Refundación del Estado en América Latina: perspectivas desde una epistemología del Sur. La Paz: Plural, 2010.

SHIZHA, Edward. Indigenous? What indigenous knowledge? Beliefs and attitudes of rural primary school teachers towards indigenous knowledge in the science curriculum in zimbabwe. Australian Journal of Indigenous Education, n. 37, p. 80-90, 2008.

ZAMBRANA, Amilcar (Ed.). Pluralismo epistemológico: reflexiones sobre la educación superior en el estado plurinacional de Bolivia. Cochabamba: Funproeib, 2014.

Recibido en: 04.10.2019

Aprobado en: 02.12.2019

Katerin Arias-Ortega es doctora en Educación. Académica del Departamento de Psicología de la Facultad de Ciencias de la Salud de la Universidad Católica de Temuco (UCT). Investigadora Joven del Centro de Investigación en Contexto Indígena e Intercultural (CIECII) de la UCT. 